PHYSICAL REVIEW D 89, 025016 (2014)

\title{
Supersymmetric gauge theories, Coulomb gases, and Chern-Simons matrix models
}

\author{
Georgios Giasemidis, ${ }^{1, *}$ Richard J. Szabo, ${ }^{2, \dagger}$ and Miguel Tierz ${ }^{3,+}$ \\ ${ }^{1}$ Rudolf Peierls Centre for Theoretical Physics, University of Oxford, 1 Keble Road, Oxford OXI 3NP, \\ United Kingdom \\ ${ }^{2}$ Department of Mathematics, Heriot-Watt University, Colin Maclaurin Building, Riccarton, \\ Edinburgh EHI4 4AS, United Kingdom, Maxwell Institute for Mathematical Sciences, Edinburgh, \\ United Kingdom, and The Tait Institute, Edinburgh, United Kingdom \\ ${ }^{3}$ Departamento de Análisis Matemático, Facultad de Ciencias Matemáticas, \\ Universidad Complutense de Madrid, Plaza de Ciencias 3, Ciudad Universitaria, 28040 Madrid, Spain
} (Received 26 October 2013; published 28 January 2014)

\begin{abstract}
We develop Coulomb gas pictures of strong and weak coupling regimes of supersymmetric Yang-Mills theory in five and four dimensions. By relating them to the matrix models that arise in Chern-Simons theory, we compute their free energies in the large $N$ limit and establish relationships between the respective gauge theories. We use these correspondences to rederive the $N^{3}$ behavior of the perturbative free energy of supersymmetric gauge theory on certain toric Sasaki-Einstein five-manifolds, and the one-loop thermal free energy of $\mathcal{N}=4$ supersymmetric Yang-Mills theory on a spatial three-sphere.
\end{abstract}

DOI: $10.1103 /$ PhysRevD.89.025016

PACS numbers: 11.10.Kk, 05.30.Fk, 11.15.Yc, 12.60.Jv

\section{INTRODUCTION AND SUMMARY}

Recent interest in six-dimensional $(2,0)$ superconformal theories [1] has been rekindled by the suggestion that the maximally supersymmetric Yang-Mills theory in five dimensions contains all degrees of freedom of the $(2,0)$ theory [2-4]. The $(2,0)$ theory lives on the boundary of $\mathrm{AdS}_{7}$, which in the Lorentzian case can be chosen to be $S^{5} \times \mathbb{R}$. The Euclidean version can have the time direction $\mathbb{R}$ compactified to a circle $S^{1}$ which reduces the dual $(2,0)$ theory to the five-dimensional supersymmetric Yang-Mills theory. In [2-4] it is argued that the Kaluza-Klein states from dimensional reduction over $S^{1}$ are mapped to instantons of the five-dimensional gauge theory.

A well-known difficulty of the six-dimensional $(2,0)$ superconformal theories is the lack of a Lagrangian description, and hence one has to use the AdS/CFT correspondence where the $(2,0)$ theories are conjectured to be dual to M-theory on an $\mathrm{AdS}_{7} \times S^{4}$ background. This supergravity dual is known to yield an $N^{3}$ growth in degrees of freedom for the free energy of the $(2,0)$ theories $[5,6]$. This dependence survives in the supergravity dual after compactification suggesting that the $N^{3}$ behavior should also appear in some way in the five-dimensional gauge theory.

In [7] the $N^{3}$ behavior is found by localization, which reduces the partition function to one that is very close to the partition function of Chern-Simons theory on $S^{3}$. In [8] the calculation of the $\mathcal{N}=1$ supersymmetric Yang-Mills

\footnotetext{
*g.giasemidis1@physics.ox.ac.uk

R.J.Szabo@hw.ac.uk

tierz@mat.ucm.es
}

partition function on $S^{5}$ is examined and, for the field theory with one adjoint hypermultiplet which in the large radius limit has an enhanced $\mathcal{N}=2$ supersymmetry, it is shown that the free energy scales as $N^{3}$ confirming the expectation from supergravity. The strong coupling limit is studied in [8] through the corresponding limit in the matrix model description, which was found by localization in [9] (based on [10]).

The partition function for the $\mathcal{N}=1$ supersymmetric gauge theory on $S^{5}$ with gauge group $U(N)$ and a massless hypermultiplet in the adjoint representation has the matrix model representation

$$
\begin{aligned}
Z_{\mathrm{YM}}^{(5)}= & \int_{\mathbb{R}^{N}} \prod_{i=1}^{N} \mathrm{~d} \phi_{i} \mathrm{e}^{-8 \pi^{3} r \varphi_{i}^{2} / g_{\mathrm{YM}}^{2}} \\
& \times \prod_{i<j}\left(\sinh \pi \phi_{i j}\right)^{2}\left(\cosh \pi \phi_{i j}\right)^{1 / 2} \\
& \times \frac{\mathcal{S}_{3}\left(\mathrm{i} \phi_{i j}\right)}{\left(\mathcal{S}_{3}\left(\frac{1}{2}+\mathrm{i} \phi_{i j}\right) \mathcal{S}_{3}\left(\frac{1}{2}-\mathrm{i} \phi_{i j}\right)\right)^{1 / 2}},
\end{aligned}
$$

where $r$ is the radius of the five-sphere $S^{5}, \phi_{i j}=\phi_{i}-\phi_{j}$ with $\phi_{i}$ dimensionless matrix eigenvalues, and $\mathcal{S}_{3}(x)$ is the triple sine function which solves the equation [11]

$$
\frac{\mathrm{d} \log \mathcal{S}_{3}(x)}{\mathrm{d} x}=\pi x^{2} \cot (\pi x) .
$$

The matrix model represents the contribution to the localization formula for the path integral around the trivial connection and hence gives the full perturbative partition function, whereas the instanton sector contributes with overall factors of order $\mathcal{O}\left(\exp \left(-16 \pi^{3} r / g_{\mathrm{YM}}^{2}\right)\right)$. 
In this paper we shall relate the strong coupling limit of (1.1) to the strong coupling expansion of the matrix model for $U(N)$ Chern-Simons gauge theory on $S^{3}$, whose partition function is given by [12]

$$
\begin{aligned}
Z_{\mathrm{CS}}= & \frac{\mathrm{e}^{-g_{\mathrm{s}} N\left(N^{2}-1\right) / 12}}{N !} \\
& \times \int_{\mathbb{R}^{N}} \prod_{i=1}^{N} \frac{\mathrm{d} u_{i}}{2 \pi} \mathrm{e}^{-u_{i}^{2} / 2 g_{\mathrm{s}}} \prod_{i<j}\left(2 \sinh \frac{u_{i}-u_{j}}{2}\right)^{2},
\end{aligned}
$$

where $g_{\mathrm{s}}$ is the string coupling constant which is related to the level $k \in \mathbb{Z}$ of the Chern-Simons gauge theory by $g_{\mathrm{s}}=2 \pi \mathrm{i} /(k+N)$; in the following we work in the analytical continuation of Chern-Simons theory with $g_{\mathrm{s}}$ real, as is done in topological string theory [13], which is the $q$ deformation of the Yang-Mills theory on $S^{2}$ [14]. This matrix model also represents the contribution of the trivial flat connection, which for the Chern-Simons gauge theory on $S^{3}$ constitutes the complete contribution to the path integral. Through this relation, we shall show how to extract the $N^{3}$ dependence of the free energy directly from the Chern-Simons matrix model using somewhat elementary techniques. This relationship has the virtue of naturally explaining certain aspects of the exact localization of the five-dimensional supersymmetric gauge theory; for example, we show that the contributions from adjoint hypermultiplets to the localization formula in five dimensions can be interpreted geometrically as a framing contribution of the three-manifold in the Chern-Simons partition function.

A key interpretation that we advocate from this relationship between the two apparently distinct gauge theories is through their natural appearances in the theory of onedimensional exactly solvable models. The partition function (1.3) of the Chern-Simons theory can be interpreted as the $\mathrm{L}^{2}$-norm of the ground state wave function of a fermionic model on a cylinder of radius $R_{c}=1$ with Hamiltonian [15]

$$
\begin{aligned}
H= & -\sum_{i=1}^{N} \frac{\partial^{2}}{\partial x_{i}^{2}}+\frac{1}{g_{\mathrm{s}}^{2}} \sum_{i=1}^{N} x_{i}^{2} \\
& +\frac{1}{g_{\mathrm{s}} R_{c}} \sum_{i<j}\left(x_{i}-x_{j}\right) \operatorname{coth}\left(\frac{x_{i}-x_{j}}{2 R_{c}}\right) .
\end{aligned}
$$

The strong coupling limit that identifies the two gauge theories is then a thin cylinder limit $R_{c} \rightarrow 0$, wherein $\operatorname{coth}\left(\frac{x_{i}-x_{j}}{2 R_{c}}\right) \rightarrow \operatorname{sgn}\left(x_{i}-x_{j}\right)$, which as we shall see also identifies the five-dimensional supersymmetric gauge theory with a one-dimensional nonrelativistic charged Bose gas. With the aid of some known Coulomb gas techniques, we are able to provide yet another derivation of the $N^{3}$ behavior of the free energy through relatively straightforward methods.
Our considerations of five-dimensional supersymmetric Yang-Mills theory are contained in Sec. II.

The wave function of the fermionic model that appears in Chern-Simons theory is the dimensional reduction of the Laughlin wave function on the cylinder, which in the quantum Hall effect is the ground state of an electron gas in two dimensions; in particular, the fermionic model lives on a longitudinal line on the surface of the cylinder. Via this observation we use Coulomb gas techniques to evaluate the one-loop thermal free energy of $\mathcal{N}=4$ supersymmetric Yang-Mills theory on $S^{3} \times S^{1}$, and reproduce the large $N$ results of [16] which interprets the gauge theory effective action as a two-dimensional Coulomb gas in an external potential; via holographic duality this finite-temperature gauge theory can be used to relate weakly coupled plasmas to black holes and to map out stringy effects on the nature of black hole physics. A thin cylinder limit brings the twodimensional and one-dimensional models together, and it has been argued that the two systems are adiabatically connected. Alternatively, by constraining electrons in a strong magnetic field to the lowest Landau level, the dimensional reduction can be achieved by taking a transversal section of the cylinder as the space variable; via Fourier transformation, the dual reduction is along a longitudinal line leading to the wave function of the fermionic model. Hence the Chern-Simons matrix model also gives a one-dimensional description of the quantum Hall effect on a cylinder. Our considerations of the fourdimensional supersymmetric Yang-Mills theory are the topic of Sec. III.

\section{II. $\mathcal{N}=1$ SUPERSYMMETRIC YANG-MILLS THEORY ON $S^{5}$}

\section{A. Strong coupling regime}

In this section we shall focus on the strong coupling limit $\lambda \rightarrow \infty$ of the supersymmetric Yang-Mills theory on $S^{5}$, where $\lambda:=g_{\mathrm{YM}}^{2} N / r$ is the 't Hooft coupling constant. In this regime the partition function (1.1) takes the form ${ }^{1}$ [8]

$$
\hat{Z}_{\mathrm{YM}}^{(5)}=\int_{\mathbb{R}^{N}} \prod_{i=1}^{N} \mathrm{~d} \phi_{i} \exp \left(-\frac{8 \pi^{3} N}{\lambda} \sum_{i=1}^{N} \phi_{i}^{2}+\frac{9 \pi}{4} \sum_{i<j}\left|\phi_{i}-\phi_{j}\right|\right) .
$$

The partition function (2.1) is studied in [8] using the saddle-point method, exhibiting the $N^{3}$ behavior of the free energy at large $N$.

On the other hand, by rescalling the variables $u_{i} \rightarrow$ $\sqrt{2 g_{\mathrm{s}}} u_{i}$ the matrix integral (1.3) in the strong coupling limit $g_{\mathrm{s}} \rightarrow \infty$ becomes

\footnotetext{
${ }^{1}$ Throughout we denote partition functions in their various limits, e.g. strong and weak coupling limits, thermodynamic limits, etc., with a hat.
} 


$$
\begin{aligned}
\hat{Z}_{\mathrm{CS}}= & \frac{\mathrm{e}^{-g_{\mathrm{s}} N\left(N^{2}-1\right) / 12}}{N !}\left(\frac{g_{\mathrm{s}}}{2 \pi^{2}}\right)^{N / 2} \\
& \times \int_{\mathbb{R}^{N}} \prod_{i=1}^{N} \mathrm{~d} u_{i} \exp \left(-\sum_{i=1}^{N} u_{i}^{2}+\sqrt{2 g_{\mathrm{s}}} \sum_{i<j}\left|u_{i}-u_{j}\right|\right)
\end{aligned}
$$

Hence the strong coupling limit of the $U(N)$ Chern-Simons matrix model on $S^{3}$ also reduces to (2.1). Since the original matrix model (1.1) can be regarded as the Chern-Simons matrix model with additional terms in the integrand and both matrix models have the same strong coupling limit, the additional terms do not contribute in the strong coupling regime. Thus the $N^{3}$ behavior of the free energy should manifest itself in the exact solution of the Chern-Simons matrix model. The $N^{3}$ dependence can indeed be seen already in the solution of the Chern-Simons matrix model with the technique of orthogonal polynomials [17]; we shall show below that the exact solution of the matrix model given in [17] contains the exact evaluation of the matrix integral (2.1).

The computation of (2.1) is also intimately related to an old statistical mechanics problem studied in detail by Baxter in 1963 [18]. While the matrix model of $U(N)$ Chern-Simons gauge theory on $S^{3}$ has an interpretation as a one-component Coulomb plasma living on the surface of a cylinder in Dyson's Coulomb gas picture of random matrix ensembles [15], the expression (2.1) is the partition function of a one-dimensional Coulomb gas known as a one-dimensional jellium model [18]: The two-body interaction $\left|x_{i}-x_{j}\right|$ is a Coulomb potential in one dimension, while $\log \sinh \left(\left|x_{i}-x_{j}\right| / 2 R_{c}\right)$ is the Coulomb potential on a cylinder of radius $R_{c}$. The strong coupling limit that maps (1.3) to (2.1) is then a thin cylinder limit $R_{c} \rightarrow 0$ in the Coulomb plasma representation.

We can then compute (2.1) in two different ways. First, we apply the methods of Baxter in [18] where the onedimensional Coulomb system with a uniform background charge distribution was studied. The second method uses the exact solution of the Chern-Simons gauge theory by examining its strong coupling limit; in the process we will specify the framing contribution contained in the matrix integral (1.3) which, in the strong coupling limit, is the leading contribution.

\section{B. One-dimensional Coulomb gas}

The partition function (2.1) is essentially a jellium model in one dimension, studied in [18]. This is a system of $N$ particles at temperature $T$ each carrying a charge $-\sigma$ on a line of length $2 L$ with particle density $\rho=$ $(N-1) / 2 L$ and a uniform positive charge distribution $\rho \sigma$ along the line. The partition function of this system is given by [18]

$$
\begin{aligned}
Z_{\mathrm{J}}^{(1)}= & \mathrm{e}^{-N\left(N^{2}-1\right) / 12 \rho} \int_{[-L, L]^{N}} \prod_{i=1}^{N} \mathrm{~d} x_{i} \\
& \times \exp \left(-\frac{2 \pi \sigma^{2}}{T}\left(\rho \sum_{i=1}^{N} x_{i}^{2}-\sum_{i<j}\left|x_{i}-x_{j}\right|\right)\right),
\end{aligned}
$$

where the first and second terms in the exponential of the integrand correspond to the charge-carrier-background interaction and charge carrier self-interactions, respectively, while the proportionality term independent of $x_{i}$ is the background-background interaction (i.e. the ground state energy).

Notice that (2.3) is directly related to (2.1) under the identifications $L=9 \lambda / 64 \pi^{2}$ and

$$
\frac{\sigma^{2}}{T}=\frac{9}{8}, \quad \rho=\frac{32 \pi^{2} N}{9 \lambda} .
$$

Therefore one can compute (2.1) following the analysis of (2.3) in [18]. We start by replacing the integral (2.1) with the integral over the chamber of eigenvalue space with $\phi_{1}>\phi_{2}>\cdots>\phi_{N}$ to get

$$
\begin{aligned}
\hat{Z}_{\mathrm{YM}}^{(5)}= & N ! \int_{-\infty}^{\infty} \mathrm{d} \phi_{1} \int_{-\infty}^{\phi_{1}} \mathrm{~d} \phi_{2} \ldots \int_{-\infty}^{\phi_{N-1}} \mathrm{~d} \phi_{N} \\
& \times \exp \left(-\frac{9 \pi}{4} \sum_{i=1}^{N}\left(\rho \phi_{i}^{2}-(N-2 i+1) \phi_{i}\right)\right) .
\end{aligned}
$$

By completing the square in the potential term and setting $v_{i}=\rho \phi_{i}+(2 i-N-1) / 2$ we find

$$
\begin{aligned}
\hat{Z}_{\mathrm{YM}}^{(5)}= & \mathrm{e}^{\frac{9 \pi}{48 \rho} N\left(N^{2}-1\right) \frac{N !}{\rho^{N}}} \int_{-\infty}^{\infty} \mathrm{d} v_{1} \int_{-\infty}^{v_{1+1}} \mathrm{~d} v_{2} \cdots \int_{-\infty}^{v_{N-1}+1} \mathrm{~d} v_{N} \\
& \times \exp \left(-\frac{9 \pi}{4 \rho} \sum_{i=1}^{N} v_{i}^{2}\right) .
\end{aligned}
$$

The multiple integral (2.6) can be computed by first using the approximation $v_{i}+1 \simeq v_{i}$ for any $i$ at large $N$, and employing the formula

$$
\begin{aligned}
& \int_{-\infty}^{v_{i-1}} \mathrm{~d} v_{i} \mathrm{e}^{-c v_{i}^{2}}\left(1+\operatorname{erf}\left(v_{i} \sqrt{c}\right)\right)^{N-i} \\
& \quad=\frac{\sqrt{\pi}}{2(N-i+1) \sqrt{c}}\left(1+\operatorname{erf}\left(v_{i-1} \sqrt{c}\right)\right)^{N-i+1}
\end{aligned}
$$

for $2 \leq i \leq N$, where $\operatorname{erf}(x):=\frac{2}{\sqrt{\pi}} \int_{0}^{x} \mathrm{~d} y \mathrm{e}^{-y^{2}}$ is the error function and $c=9 \pi / 4 \rho$ is a constant. The final integration over $v_{1}$ yields

$$
\int_{-\infty}^{\infty} \mathrm{d} v_{1} \mathrm{e}^{-c v_{i}^{2}}\left(1+\operatorname{erf}\left(v_{1} \sqrt{c}\right)\right)^{N-1}=\frac{2^{N-1} \sqrt{\pi}}{N \sqrt{c}}
$$

and whence the partition function (2.6) takes the form 


$$
\begin{aligned}
\hat{Z}_{\mathrm{YM}}^{(5)} & =\frac{\mathrm{e}^{\frac{9 \pi}{48 \rho} N\left(N^{2}-1\right)}}{\rho^{N}} N !\left(\sqrt{\frac{\pi}{c}}\right)^{N-1}\left(\prod_{i=2}^{N} \frac{1}{2(N-i+1)}\right) \frac{2^{N-1} \sqrt{\pi}}{N \sqrt{c}} \\
& =\mathrm{e}^{\frac{9 \pi}{48 \rho} N\left(N^{2}-1\right)}\left(\frac{4}{9 \rho}\right)^{N / 2}
\end{aligned}
$$

for $c=9 \pi / 4 \rho$. The free energy of the strong coupling regime of the supersymmetric gauge theory on $S^{5}$ at large $N$ is therefore given by

$$
\hat{F}_{\mathrm{YM}}^{(5)}=-\log \hat{Z}_{\mathrm{YM}}^{(5)}=-\frac{27}{512} \frac{g_{\mathrm{YM}}^{2}}{\pi r} N^{3},
$$

in agreement with the result of [8].

\section{Chern-Simons matrix model}

The matrix integral in (2.2) is of the form (2.1): By rescaling the eigenvalues $\phi_{i} \rightarrow \sqrt{\lambda / 8 \pi^{3} N} \phi_{i}$ in (2.1) and identifying the large couplings as

$$
g_{\mathrm{s}}=\frac{81 \lambda}{256 \pi N}
$$

we have explicitly

$$
\hat{Z}_{\mathrm{YM}}^{(5)}=\left(\frac{8}{9}\right)^{N} N ! \mathrm{e}^{g_{\mathrm{s}} N\left(N^{2}-1\right) / 12} \hat{Z}_{\mathrm{CS}} .
$$

The matrix model (1.3) was solved in [17] via the Stieltjes-Wigert orthogonal polynomials, and its exact solution takes the form

$$
Z_{\mathrm{CS}}=\left(\frac{g_{\mathrm{s}}}{2 \pi}\right)^{N / 2} \mathrm{e}^{g_{\mathrm{s}} N\left(N^{2}-1\right) / 12} \prod_{j=1}^{N}\left(1-q^{j}\right)^{N-j},
$$

where $q=\mathrm{e}^{-g_{\mathrm{s}}}$. Applying the identification (2.11) we now write (2.12) as

$$
\hat{Z}_{\mathrm{YM}}^{(5)}=N !\left(\frac{\lambda}{8 \pi^{2} N}\right)^{N / 2} \mathrm{e}^{\frac{27 \lambda}{512 \pi}\left(N^{2}-1\right)} \lim _{q \rightarrow 0} \prod_{j=1}^{N}\left(1-q^{j}\right)^{N-j}
$$

The limit $q \rightarrow 0$ in (2.14), called the crystal limit in quantum group theory [19], can easily be computed following [20]. For this, we take $\tilde{\lambda}=g_{\mathrm{s}} N$ constant and consider

$$
P(N, \tilde{\lambda}):=N \sum_{j=1}^{N}\left(1-\frac{j}{N}\right) \log \left(1-\mathrm{e}^{-\tilde{\lambda}^{\frac{j}{N}}}\right) .
$$

This expression is a Riemann sum over $y_{j}=\frac{j}{N}$ with $y_{j}-$ $y_{j-1}=\frac{1}{N}$ for $j=1, \ldots ., N$. Since $\frac{1}{N} \leq y_{j} \leq 1-\frac{1}{N}$, in the large $N$ limit we can write it as the integral

$$
P(N, \tilde{\lambda})=N^{2} \int_{0}^{1} \mathrm{~d} y(1-y) \log \left(1-\mathrm{e}^{-\tilde{\lambda} y}\right)
$$

$\underset{\sim}{\text { and }}$ an additional change of variables $x=\tilde{\lambda} y$ in the limit $\tilde{\lambda} \rightarrow \infty$ gives finally

$\hat{P}(N, \tilde{\lambda})=\frac{N^{2}}{\tilde{\lambda}} \int_{0}^{\infty} \mathrm{d} x \log \left(1-\mathrm{e}^{-x}\right)=-\frac{128 \pi^{3} N^{2}}{243 \lambda}$.

It follows that

$$
\begin{aligned}
\hat{Z}_{\mathrm{YM}}^{(5)}= & \left(\frac{g_{\mathrm{YM}}^{2}}{4 \pi^{2} r}\right)^{N / 2} N ! \exp \left(\frac{27}{512} \frac{g_{\mathrm{YM}}^{2}}{\pi r} N\left(N^{2}-1\right)\right. \\
& \left.-\frac{128}{243} \frac{\pi^{3} r}{g_{\mathrm{YM}}^{2}} N\right)
\end{aligned}
$$

and the free energy at leading order in $N$ is given by

$$
\hat{F}_{\mathrm{YM}}^{(5)}=-\frac{27}{512} \frac{g_{\mathrm{YM}}^{2}}{\pi r} N^{3}
$$

which coincides with (2.10).

\section{Framing}

The nontrivial part of the supersymmetric gauge theory partition function on $S^{5}$, given by the product term in the Chern-Simons partition function (2.13), is subleading in $N$ and does not appear in the final result of (2.19). This naturally leads us into a discussion of the framing contribution in Chern-Simons theory and how it is represented by the matrix models.

Chern-Simons gauge theory is a theory of framed knots and links [21]. For gauge group $G=U(N)$, the contribution of a framing $\Pi_{s}$ on the three-sphere $S^{3}$ is parametrized by an integer $s \in \mathbb{Z}$ and takes the form [22]

$$
\delta\left(\Pi_{s}\right)=\mathrm{e}^{2 \pi \mathrm{i} s c / 24},
$$

where $c=k \operatorname{dim}(G) /(k+N)$ is the central charge of the Wess-Zumino-Witten conformal field theory based on the affine extension of $G$. The central charge can be expressed in terms of the Weyl vector $\rho$ of the gauge group and one has [22]

$$
\delta\left(\Pi_{s}\right)=\mathrm{e}^{\pi \mathrm{i} s|\rho|^{2} k / N(k+N)}=\mathrm{e}^{\pi \mathrm{i} s|\rho|^{2} / N} \mathrm{e}^{-g_{\mathrm{s}} s|\rho|^{2} / 2},
$$

where we used the identification $g_{\mathrm{s}}=2 \pi \mathrm{i} /(k+N)$ and

$$
|\rho|^{2}=\frac{1}{24} N\left(N^{2}-1\right)
$$

The inclusion of framing modifies the Chern-Simons partition function by rescaling it with the phase (2.20), and one can therefore consider a family of partition functions $Z_{\mathrm{CS}}^{s}$ parametrized by $s \in \mathbb{Z}$ with

$$
Z_{\mathrm{CS}}^{s}=\delta\left(\Pi_{s}\right) Z_{\mathrm{CS}}^{0},
$$


where the partition function of the Chern-Simons theory in the canonical framing $s=0$ on $S^{3}$ is given by

$$
Z_{\mathrm{CS}}^{0}=\left(\frac{g_{\mathrm{s}}}{2 \pi}\right)^{N / 2} N ! \prod_{j=1}^{N}\left(1-q^{j}\right)^{N-j} .
$$

Thus the partition function (1.3) carries a nontrivial framing dependence, as is evident by comparing (2.24) with (2.13); precisely, the Hermitian matrix model formulation of the Chern-Simons gauge theory on $S^{3}$ carries a framing contribution (2.20) with $s=-4$ such that

$$
Z_{\text {CS }}=\mathrm{e}^{-\pi \mathrm{i}\left(N^{2}-1\right) / 6} Z_{\text {CS }}^{s=-4} \text {. }
$$

Let us consider now the strong coupling limit $g_{\mathrm{s}} \rightarrow \infty$. In this regime the five-dimensional supersymmetric gauge theory on the boundary is related to the $q \rightarrow 0$ limit of the analytically continued Chern-Simons theory with some framing contribution, through the respective matrix integral formulations. Notice that in this strong coupling limit, the framing dependence in the Chern-Simons theory alters the prefactor of the leading term in $N$, which is dominant in the limit. Therefore the $N^{3}$ behavior of the $q \rightarrow 0$ limit of the Chern-Simons theory comes from the framing term, as the nontrivial product factor is subleading.

In fact, we can show that there exists an appropriate framing which explains the discrepancy between the gauge theory and gravity results. The Yang-Mills free energy from the matrix model computation is given by (2.19), while the classical supergravity action in the $\mathrm{AdS}_{7}$ background receives contributions from the bulk, the boundary, and the regularization counterterms, and is given by [8]

$$
\hat{F}_{\text {grav }}=-\frac{5 \pi R_{6}}{12 r} N^{3},
$$

where $R_{6}$ is the radius of the compactification circle $S^{1}$ on the boundary. The Kaluza-Klein modes from compactification on $S^{1}$ are mapped to instantons of the five-dimensional gauge theory, which suggests the identification [2-4]

$$
R_{6}=\frac{g_{\mathrm{YM}}^{2}}{8 \pi^{2}}
$$

leading to

$$
\hat{F}_{\text {grav }}=-\frac{5}{96} \frac{g_{\mathrm{YM}}^{2}}{\pi r} N^{3} .
$$

The mismatch in the numerical prefactors is restored by multiplying the partition function (2.18) of the boundary supersymmetric gauge theory with an extra factor to give the partition function of a boundary theory (that we denote by $\hat{Z}_{\mathrm{B}}$ ) which has the form

$$
\hat{Z}_{\mathrm{B}}=\mathrm{e}^{-\frac{1}{1536} g_{\mathrm{YM}}^{2} \pi N^{3}} \hat{Z}_{\mathrm{YM}}^{5}=\left(\frac{8}{9}\right)^{N} N ! \hat{Z}_{\mathrm{CS}}^{s=-8} \mathrm{e}^{\mathcal{O}\left(N^{2}\right)}
$$

and the required framing parameter is $s=-640 / 81 \simeq-8$. Alternatively, since the leading term in the $q \rightarrow 0$ limit comes from the second exponential of the framing in (2.21), we equate

$$
\exp \left(\frac{5 g_{\mathrm{YM}}^{2}}{96 \pi r} N^{3}\right)=\exp \left(-\frac{g_{\mathrm{s}} s N\left(N^{2}-1\right)}{48}\right)
$$

and by taking into account the identification (2.11) we find that the boundary partition function can be expressed as the strong coupling limit of the framed Chern-Simons partition function $Z_{\mathrm{CS}}^{s}$ with framing parameter

$$
s=-\frac{5 \cdot 256 \cdot 48}{96 \cdot 81}=-\frac{640}{81} \simeq-8 .
$$

\section{E. Massive hypermultiplet}

It was shown in [8] that the $N^{3}$ behavior in the gauge theory on $S^{5}$ originates from the presence of a single massless adjoint hypermultiplet, where the field theory has only $\mathcal{N}=1$ supersymmetry. In the case of a massive adjoint hypermultiplet, it was argued in [7] that the global symmetry is enhanced at a point where the mass is $M=\frac{1}{2 r}$. Then the massless case can be thought of as a deformation of the flat space theory by the radius parameter $r$, which in the large radius limit has an enhanced $\mathcal{N}=2$ supersymmetry. The massive case is considered in [23], where it was shown that the mass parameter enters into the numerical prefactor of the free energy (2.19). In particular, the strong coupling limit of the partition function becomes

$$
\begin{aligned}
\hat{Z}_{\mathrm{YM}}^{(5)}(m)= & \int_{\mathbb{R}^{N}} \prod_{i=1}^{N} \mathrm{~d} \phi_{i} \exp \left(-\frac{8 \pi^{3} N}{\lambda} \sum_{i=1}^{N} \phi_{i}^{2}\right. \\
& \left.+\pi\left(\frac{9}{4}+m^{2}\right) \sum_{i<j}\left|\phi_{i}-\phi_{j}\right|\right),
\end{aligned}
$$

where $m=-\mathrm{i} M r$ is the mass rotated to the imaginary axis, a step required for the localization of the path integral. Hence the free energy (2.19) is modified to

$$
\hat{F}_{\mathrm{YM}}^{(5)}=-\left(\frac{9}{4}+m^{2}\right)^{2} \frac{g_{\mathrm{YM}}^{2}}{96 \pi r} N^{3}
$$

There are now two key observations [23]. First, the matching of the supersymmetric Wilson loop that wraps the five-sphere $S^{5}$ at strong coupling with the regularized circular Wilson loop in supergravity suggests the new identification

$$
R_{6}=\frac{5 g_{\mathrm{YM}}^{2}}{32 \pi^{2}},
$$

in contrast to (2.27) which led to (2.28). Second, one has to rotate back to real values of the mass parameter, so that 
$m=\frac{1}{2}$ at the enhancement point. This results in agreement between the free energy of the five-dimensional supersymmetric Yang-Mills theory and of its supergravity dual.

By suitably modifying the identifications of parameters in (2.4) and (2.11), one can easily obtain (2.33) via both the one-dimensional Coulomb gas picture of Sec. II B and the strong coupling regime of the Chern-Simons theory from Sec. II C. In the Chern-Simons description the $N^{3}$ behavior originates from the framing contribution whose choice controls the prefactor containing the $N^{3}$ dependence, while from the point of view of the supersymmetric gauge theory the $N^{3}$ dependence comes from the presence of a single hypermultiplet whose mass parameter controls the prefactor of the free energy. Applying the arguments of Sec. II D we should now equate

$$
\exp \left(\frac{25 g_{\mathrm{YM}}^{2}}{384 \pi r} N^{3}\right)=\exp \left(-\frac{g_{\mathrm{s}} s N\left(N^{2}-1\right)}{48}\right),
$$

where the string coupling identification (2.11) must be modified to $g_{\mathrm{s}}=\frac{25 \lambda}{64 \pi N}$ in order to accommodate the dependence on the mass parameter $m=\frac{1}{2}$. This yields the framing parameter $s=-8$. This is now an exact integer result, and it demonstrates the agreement of the strong coupling regime of the Chern-Simons theory with the framing contribution $s=-8$ and the large $N$ supergravity dual under the identification (2.34). This consistency between the Chern-Simons gauge theory on $S^{3}$ and the supersymmetric Yang-Mills theory on $S^{5}$ raises the intriguing possibility that there might be a deeper geometric connection between the adjoint hypermultiplet of the five-dimensional gauge theory and the framing contribution in Chern-Simons theory.

\section{F. Lens space matrix models}

For completeness, let us now generalize our computations to the strong coupling regime $g_{\mathrm{s}} \rightarrow \infty$ of the Chern-Simons theory on lens spaces $L(P, Q)$, studied in [12,24]. The contribution of the trivial flat connection to the path integral of this gauge theory is described by the matrix model

$$
\begin{aligned}
Z_{C S}^{P, Q}= & \int_{\mathbb{R}^{N}} \prod_{i=1}^{N} \frac{\mathrm{d} u_{i}}{2 \pi} \mathrm{e}^{-u_{i}^{2} / 2 g_{\mathrm{s}}} \prod_{i<j}\left(2 \sinh \left(\frac{u_{i}-u_{j}}{2 P}\right)\right) \\
& \times\left(2 \sinh \left(\frac{u_{i}-u_{j}}{2 Q}\right)\right),
\end{aligned}
$$

where $P$ and $Q$ are coprime integers. For $P=Q=1$ this matrix integral is related to the partition function (1.3) of $U(N)$ Chern-Simons theory on $S^{3}$ as

$$
Z_{\mathrm{CS}}=\frac{\mathrm{e}^{-g_{\mathrm{s}} N\left(N^{2}-1\right) / 12}}{N !} Z_{\mathrm{CS}}^{1,1}
$$

The matrix integral (2.36) is computed exactly in [24] via biorthogonal Stieltjes-Wigert polynomials with the result

$$
\begin{aligned}
Z_{\mathrm{CS}}^{P, Q}= & N !\left(\frac{g_{\mathrm{s}}}{2 \pi}\right)^{N / 2} \exp \left(\frac { g _ { \mathrm { s } } } { 2 P ^ { 4 } } N \left[-\left(1+\frac{1}{2}\left(1+\frac{P}{Q}\right)\right.\right.\right. \\
& \left.\left.\times(N-1))^{2}+1+\frac{4}{3}\left(N^{2}-1\right)\right]\right) \\
& \times \prod_{j=1}^{N}\left(1-\bar{q}^{j / P Q}\right)^{N-j},
\end{aligned}
$$

where $\bar{q}=\mathrm{e}^{-g_{\mathrm{s}} / P^{2}}$. We have already seen that in the case of the three-sphere $P=Q=1$ the product contributes subleading terms of order $N$ to the free energy in the limit $g_{\mathrm{s}} \rightarrow \infty$. The situation is the same for generic finite integers $P, Q$, and therefore the leading $N^{3}$ behavior comes from the exponential in the expression (2.38). The corresponding free energy $\hat{F}_{\mathrm{CS}}^{P, Q}=-\log \hat{Z}_{\mathrm{CS}}^{P, Q}$ at large $N$ is given by

$$
\hat{F}_{\mathrm{CS}}^{P, Q}=-\frac{g_{\mathrm{s}}}{2 P^{4}}\left(\frac{13}{12}-\frac{P}{2 Q}\left(1+\frac{P}{2 Q}\right)\right) N^{3} .
$$

Following the analogous manipulations for the $S^{3}$ matrix model, the strong coupling limit of (2.36) takes the form

$$
\begin{aligned}
\hat{Z}_{\mathrm{CS}}^{P, Q}= & \left(\frac{g_{\mathrm{s}}}{2 \pi^{2}}\right)^{N / 2} \int_{\mathbb{R}^{N}} \prod_{i=1}^{N} \mathrm{~d} u_{i} \exp \left(-\sum_{i=1}^{N} u_{i}^{2}\right. \\
& \left.+\sqrt{\frac{g_{\mathrm{s}}}{2}} \alpha \sum_{i<j}\left|u_{i}-u_{j}\right|\right),
\end{aligned}
$$

where $\alpha=\frac{1}{P}+\frac{1}{Q}$. It is tempting to compare this partition function with that of the supersymmetric Yang-Mills theory on the squashed toric Sasaki-Einstein five-manifolds $Y^{P, Q}$ [25] which was studied in [26]. In the limit of strong 't Hooft coupling $\lambda=g_{\mathrm{YM}}^{2} N / r$, the equivariant perturbative partition function for gauge group $U(N)$ and a massless matter hypermultiplet in the adjoint representation simplifies to the matrix model

$$
\begin{aligned}
\hat{Z}_{\mathrm{YM}}^{(5)}(P, Q)= & \int_{\mathbb{R}^{N}} \prod_{i=1}^{N} \mathrm{~d} \phi_{i} \exp \left(-\frac{8 \pi^{3} N \varrho}{\lambda} \sum_{i=1}^{N} \phi_{i}^{2}\right. \\
& \left.+\frac{\pi \varrho}{4}\left(\sum_{l=1}^{4} \omega_{l}\right)^{2} \sum_{i<j}\left|\phi_{i}-\phi_{j}\right|\right),
\end{aligned}
$$

where $Q$ is the ratio of the equivariant volume of $Y^{P, Q}$ to the volume of $S^{5}$ and $\omega_{1}, \omega_{2}, \omega_{3}, \omega_{4}$ are equivariant parameters for the isometric action of $U(1)^{4}$ on $\mathbb{C}^{4}$. Then (2.41) is proportional to (2.40) under the identification of the parameters

$$
\frac{\varrho}{128}\left(\sum_{l=1}^{4} \omega_{l}\right)^{4} \frac{\lambda}{\pi N}=\frac{g_{\mathrm{s}} \alpha^{2}}{2} .
$$

Using the identification (2.42) we can then write the strong coupling free energy as

$$
\hat{F}_{\mathrm{CS}}^{P, Q}=-f(P, Q)\left(\sum_{l=1}^{4} \omega_{l}\right)^{4} \varrho \frac{g_{\mathrm{YM}}^{2}}{\pi r} N^{3},
$$


where

$$
f(P, Q)=\frac{1}{128 \alpha^{2} P^{4}}\left(\frac{13}{12}-\frac{P}{2 Q}\left(1+\frac{P}{2 Q}\right)\right) .
$$

For $P=Q=1$ we get $f(1,1)=1 / 1536$, which agrees with the result of [26]; this corroborates the surprising universality of the $N^{3}$ behavior of the perturbative free energy on all fivemanifolds $Y^{P, Q}$ that were observed in [26]. The more general $L(P, Q)$ matrix models may be related to a localization calculation of the five-dimensional supersymmetric YangMills theory on the Sasaki-Einstein spaces $L^{a, b, c}$ which generalize $Y^{P, Q}$, but such a calculation is currently lacking in the literature and is hence left for future work.

\section{G. One-dimensional wave functions}

The partition functions of some gauge theories on $S^{3}$ can be written as the norm or the overlap of some onedimensional quantum mechanical wave functions. This is true of the $\mathcal{N}=4$ theories on $S^{3}$ that arise as the lowenergy limit of $\mathcal{N}=4$ supersymmetric Yang-Mills theory in four dimensions [27], which is also connected to the six-dimensional $(2,0)$ superconformal theories via certain dimensional reductions. It is also the case of the ChernSimons gauge theory [15].

For the one-dimensional wave function

$$
\Psi_{0}\left(x_{1}, \ldots, x_{N}\right)=\prod_{i=1}^{N} \mathrm{e}^{-\frac{\omega}{2} x_{i}^{2}} \prod_{i<j} \exp \left(\frac{c\left|x_{i}-x_{j}\right|}{2}\right)
$$

the same direct approach in [15] can be used to find the general Hamiltonian of a bosonic model for which (2.45) is a ground state. It can also be found as a limit of the Hamiltonian for the Chern-Simons fermionic model (1.4) which in its most general version is characterized by a ground state wave function [15]

$\Psi_{0}^{(m)}\left(x_{1}, \ldots, x_{N}\right)=\prod_{i=1}^{N} \mathrm{e}^{-x_{i}^{2} / 2 g_{\mathrm{s}}} \prod_{i<j}\left(\sinh \frac{x_{i}-x_{j}}{2 R_{c}}\right)^{m}$,

where $m$ is a positive parameter. The corresponding Hamiltonian is

$$
\begin{aligned}
H_{m}= & -\sum_{i=i}^{N} \frac{\partial^{2}}{\partial x_{i}^{2}}+\frac{1}{g_{\mathrm{s}}^{2}} \sum_{i=1}^{N} x_{i}^{2} \\
& +\frac{m}{g_{\mathrm{s}} R_{c}} \sum_{i<j}\left(x_{i}-x_{j}\right) \operatorname{coth}\left(\frac{x_{i}-x_{j}}{2 R_{c}}\right) \\
& +\frac{m(m-1)}{2 R_{c}} \sum_{i<j} \frac{1}{\sinh ^{2}\left(\frac{x_{i}-x_{j}}{2 R_{c}}\right)} .
\end{aligned}
$$

For $m=1$ we obtain the Hamiltonian $H=H_{1}$ in (1.4). The bosonic and fermionic models are related through the limit
$R_{c} \rightarrow 0$, which as discussed earlier is a thin cylinder limit. First, let us see what happens to the two-body term of the wave function in the limit

$$
\begin{aligned}
\lim _{R_{c} \rightarrow 0}\left(\sinh \frac{x_{i}-x_{j}}{2 R_{c}}\right)^{m}= & 2^{-m}\left(\operatorname{sgn}\left(x_{i}-x_{j}\right)\right)^{m} \\
& \times \exp \left(\frac{m\left|x_{i}-x_{j}\right|}{2 R_{c}}\right) .
\end{aligned}
$$

If $m$ is even, the sign term does not appear. Having $m$ odd and keeping the term $\operatorname{sgn}\left(x_{i}-x_{j}\right)$ can be interpreted as a fermionization of the resulting boson wave function, in the sense of [28]. We can now identify $c=\frac{m}{R_{c}}$ with the usual parameter of the Lieb-Liniger model [29]. To obtain generic values of $c$ in the thin cylinder limit, we need to take $m \rightarrow 0$, in which case the sign terms above disappear. Thus in the limit $R_{c} \rightarrow 0$ the wave function of the fermionic model (2.46) reduces to (2.45) (up to normalization) with $\omega=\frac{1}{g_{\mathrm{s}}}$ and $c=\frac{m}{R_{c}}$. The Hamiltonian (2.47) correspondingly becomes

$$
\begin{aligned}
H_{0}= & -\sum_{i=i}^{N} \frac{\partial^{2}}{\partial x_{i}^{2}}+\frac{1}{g_{\mathrm{s}}^{2}} \sum_{i=1}^{N} x_{i}^{2}+\frac{c}{g_{s}} \sum_{i<j}\left|x_{i}-x_{j}\right| \\
& +4 c(m-1) \sum_{i<j} \delta\left(x_{i}-x_{j}\right) .
\end{aligned}
$$

The Hamiltonian (2.49) can be regarded as a generalization of the Lieb-Liniger model [29], although the special case that appears in Chern-Simons theory is (2.46) with $m=1$. Therefore, in the limit considered above, it leads to the charged Bose gas without delta-function interactions. For this model the Coulomb gas interpretation holds for both the Hamiltonian and the Dyson Coulomb gas picture of the wave function, since both cases involve the onedimensional Coulomb potential $\left|x_{i}-x_{j}\right|$.

\section{III. $\mathcal{N}=4$ SUPERSYMMETRIC YANG-MILLS THEORY ON $S^{3} \times S^{1}$}

\section{A. Weak coupling regime}

We shall focus now on $\mathcal{N}=4$ supersymmetric YangMills theory on $S^{3} \times S^{1}$ and its representation as a Coulomb gas on $\mathbb{R} \times S^{1}$ at weak (but finite) 't Hooft coupling $\lambda=g_{\mathrm{YM}}^{2} N$; the radius of $S^{3}$ is denoted $R$, and the inverse radius of $S^{1}$ is the temperature $T$. Based on the one-loop determinants computed in [30], the effective action in the low temperature limit $R T \ll 1$ is shown by [16] to become

$$
\begin{aligned}
\hat{S}_{\mathrm{YM}}^{(4)}[z, \bar{z}]= & N^{2}\left(\frac{3 \beta}{16 R}-\log 2\right)+\frac{N \pi^{2} R}{\beta \lambda} \sum_{i=1}^{N}\left(z_{i}+\bar{z}_{i}\right)^{2} \\
& -2 \sum_{i<j} \log \left|\sinh \left(z_{i}-z_{j}\right)\right|
\end{aligned}
$$


where $\beta=\frac{1}{T}$ while $z_{i}=\frac{1}{2}\left(\beta \phi_{i}+\mathrm{i} \theta_{i}\right)$ are complex scalar fields with $\phi_{i}$ and $\theta_{i}$ the eigenvalues of the adjoint scalar fields and of the temporal component of the gauge field, respectively. The action (3.1) describes a Coulomb gas on a cylinder of radius $R_{c}=\frac{1}{2}$.

The Coulomb gas on the cylinder is intimately related to the Coulomb gas in one dimension, as has been studied in detail in $[31,32,33,34,35,36]$, often in the context of the corresponding quantum Hall effect on the cylinder. In particular, it is found that for certain Hamiltonians a quantum Hall ground state does not undergo a phase transition when the two-dimensional surface of the system is deformed in a quasi-one-dimensional (thin cylinder) limit $[32,33,34,35]$. Hence the two-dimensional and onedimensional systems are argued to be adiabatically connected. Recall that the $U(N)$ Chern-Simons matrix model on $S^{3}$ admits an interpretation as a Coulomb system of restricted dimension; i.e. its interaction is the Coulomb interaction on the cylinder, but the particles live in one dimension (a longitudinal line on the surface of the cylinder) [15]. In fact, the charge-density wave behavior of the Laughlin wave function on the cylinder [31] is also manifest in the oscillatory behavior of the density of states of the Chern-Simons matrix model [37]. The same property is analyzed more rigorously in [36] as an example of translational symmetry breaking. Either by dimensional reduction (which is achieved as usual by projecting to the lowest Landau level with a limit of large magnetic field $B \rightarrow \infty$ ) or by a thin cylinder limit, the two Coulomb gas descriptions are directly related. This suggests a relationship between the respective gauge theories, even though the nature of their Coulomb gas description is rather different. In the case of Chern-Simons gauge theory the matrix model arises exactly due to a localization of the path integral on flat connections [38], whereas in the case of $\mathcal{N}=4$ supersymmetric Yang-Mills theory it follows from an effective field theory approach together with a number of simplifications such as the description of the condensate of the scalar fields in a single coordinate [16].

\section{B. Two-dimensional Coulomb gas}

The corresponding partition function takes the form

$$
\begin{aligned}
\hat{Z}_{\mathrm{YM}}^{(4)}:= & \int_{\left(\mathbb{R} \times S^{1}\right)^{N}} \prod_{i=1}^{N} \mathrm{~d}^{2} z_{i} \mathrm{e}^{-\hat{S}_{\mathrm{YM}}^{(4)}[z, \bar{z}]} \\
= & \left(\frac{2^{N+1}}{\beta}\right)^{N} \mathrm{e}^{-3 \beta N^{2} / 16 R} \int_{\mathbb{R}^{N}} \prod_{i=1}^{N} \mathrm{~d} x_{i} \\
& \times \int_{[0, \pi]^{N}} \prod_{i=1}^{N} \mathrm{~d} y_{i} \mathrm{e}^{-\tau x_{i}^{2}} \prod_{i<j}\left|\sinh \left(z_{i}-z_{j}\right)\right|^{2},
\end{aligned}
$$

where $z_{i}=x_{i}+\mathrm{i} y_{i}$ are coordinates on the cylinder and we set $\tau:=4 \pi^{2} N R / \beta \lambda$ for brevity. The product in the integrand can be written as $\prod_{i<j}\left|\sinh \left(z_{i}-z_{j}\right)\right|^{2}=2^{-N(N-1)} \prod_{i=1}^{N} \mathrm{e}^{-2(N-1) x_{i}} \prod_{j<k}\left|\mathrm{e}^{2 z_{j}}-\mathrm{e}^{2 z_{k}}\right|^{2}$.

We now complete the square in the exponential in $x_{i}$ and shift variables $x_{i} \rightarrow x_{i}-(N-1) / \tau$, which implies $z_{i} \rightarrow z_{i}-(N-1) / \tau$, and then rescale $z_{i} \rightarrow z_{i} / \sqrt{\tau}$ so that the partition function finally takes the form

$$
\begin{aligned}
\hat{Z}_{\mathrm{YM}}^{(4)}= & \left(\frac{4}{\beta \tau}\right)^{N} \mathrm{e}^{-3 \beta N^{2} / 16 R} \mathrm{e}^{-N(N-1)^{2} / \tau} \\
& \times \int_{\mathbb{R}^{N}} \prod_{i=1}^{N} \mathrm{~d} x_{i} \int_{[0, \sqrt{\tau} \pi]^{N}} \prod_{i=1}^{N} \mathrm{~d} y_{i} \mathrm{e}^{-x_{i}^{2}} \\
& \times \prod_{i<j}\left|\mathrm{e}^{2 z_{i} / \sqrt{\tau}}-\mathrm{e}^{2 z_{j} / \sqrt{\tau}}\right|^{2} .
\end{aligned}
$$

\section{Laughlin wave function}

It is possible to analyze the partition function in the same spirit as in Sec. II G by using known properties of the Laughlin wave function on the cylinder. The Laughlin wave function is the ground state wave function of a two-dimensional electron gas in a uniform neutralizing background with a uniform magnetic field; it was introduced to describe the fractional quantum Hall effect [39]. The Laughlin wave function for the cylinder was first considered in [40] but did not become an object of further study until later on, beginning with [31]; mathematical aspects, such as its translational symmetry breaking, were studied in [36]. It takes the form

$$
\begin{aligned}
\Psi_{N}\left(z ; \gamma_{B}, p\right):= & \frac{\mathrm{e}^{-p^{2} \gamma_{B}^{2} N(N-1)(2 N-1) / 12}}{\sqrt{N !}}\left(\frac{\gamma_{B}}{2 \pi^{3 / 2}}\right)^{N / 2} \\
& \times \prod_{i=1}^{N} \mathrm{e}^{-x_{i}^{2} / 2} \prod_{j<k}\left(\mathrm{e}^{\gamma_{B} z_{j}}-\mathrm{e}^{\gamma_{B} z_{k}}\right)^{p},
\end{aligned}
$$

where $z_{i}=x_{i}+\mathrm{i} y_{i}$ represents the coordinates of the fermions on the cylinder, $p$ is the filling fraction of the quantum Hall system, and $\gamma_{B}$ is a dimensionful parameter defined as the ratio of the magnetic length $\ell_{B}=(\hbar / e B)^{1 / 2}$ (here set equal to 1 ) to the radius of the cylinder (here $R_{c}=\frac{1}{2}$ ). Its $\mathrm{L}^{2}$-norm is given by

$$
\begin{aligned}
C_{N}\left(\gamma_{B}, p\right):=\left\|\Psi_{N}\right\|_{2}^{2}= & \int_{\mathbb{R}^{N}} \prod_{i=1}^{N} \mathrm{~d} x_{i} \int_{\left[0,2 \pi / \gamma_{B}\right]^{N}} \\
& \times \prod_{i=1}^{N} \mathrm{~d} y_{i}\left|\Psi_{N}\left(z ; \gamma_{B}, p\right)\right|^{2} .
\end{aligned}
$$

We now notice that the gauge theory partition function (3.4) can be expressed in terms of the normalization constant $C_{N}\left(\gamma_{B}, p\right)$ for $\gamma_{B}=\frac{2}{\sqrt{\tau}}$ and $p=1$ as 


$$
\begin{aligned}
\hat{Z}_{\mathrm{YM}}^{(4)}= & \left(\frac{4 \pi^{3 / 2}}{\beta \sqrt{\tau}}\right)^{N} N ! \mathrm{e}^{-3 \beta N^{2} / 16 R} \mathrm{e}^{N\left(N^{2}-1\right) / 3 \tau} \\
& \times C_{N}\left(\gamma_{B}=\frac{2}{\sqrt{\tau}}, p=1\right) .
\end{aligned}
$$

For $p=1$ the $\mathrm{L}^{2}$-norm is given by $^{2}[36]$

$$
C_{N}\left(\gamma_{B}, p=1\right)=1 \text {. }
$$

By substituting back $\tau=4 \pi^{2} N R / \beta \lambda$ the free energy at large $N$ is thus given by

$$
\hat{F}_{\mathrm{YM}}^{(4)}=-\log \hat{Z}_{\mathrm{YM}}^{(4)}=\left(\frac{3}{16}-\frac{\lambda}{12 \pi^{2}}\right) \frac{N^{2} \beta}{R},
$$

in agreement with the calculation of the free energy given in [16] in the Coulomb gas description.

\section{Jellium on the cylinder}

The partition function (3.4) can also be computed exactly by mapping the problem to a one-component plasma on the cylinder, known as the two-dimensional jellium model, at the fermion coupling $\Gamma=2 \gamma$ which was studied in [41] for $\gamma=1$ and in [42] for arbitrary integer values of $\gamma$. The twodimensional jellium model is defined as follows. Consider $N$ particles of charge $q$ on a cylinder of radius $R_{c}$ and finite length $L$ embedded in a homogeneous background of charge density $\rho_{b}=-q n$, where $n=N /\left(2 \pi L R_{c}\right)$ so that the system remains neutral. The partition function takes the form [42]

$$
Z_{\mathrm{J}}^{(2)}=\frac{1}{N !} \int_{\Lambda^{N}} \prod_{i=1}^{N} \mathrm{~d}^{2} z_{i} \mathrm{e}^{-\beta E_{N}[z, \bar{z}]}
$$

where $\Lambda=\left[-\frac{L}{2}, \frac{L}{2}\right] \times[-\pi, \pi]$ is the cylinder and the total energy of the system is given by

$E_{N}[z, \bar{z}]=\pi n q^{2} \sum_{i=1}^{N} x_{i}^{2}-q^{2} \sum_{i<j} \log \left|2 \sinh \frac{z_{i}-z_{j}}{2 R_{c}}\right|+B_{N}$.

The first and second sums correspond to the charge-carrier - background and charge-carrier-charge-carrier interactions, respectively, while the third term $B_{N}$ which is independent of $z_{i}$ corresponds to the background-background interaction. The fermion coupling is defined as the dimensionless combination $\Gamma=\beta q^{2}$, and after some simple algebra analogous to that of Sec. III B the partition function is written as

\footnotetext{
${ }^{2}$ For $p=1$ the Laughlin wave function becomes a Slater determinant, which is the wave function of $N$ fermions. Then $C_{N}=\left\|\Psi_{N}\right\|_{2}^{2}=1$ is the normalization of the wave function of $N$ electrons.
}

$$
Z_{\mathrm{J}}^{(2)}=\frac{1}{N !} \int_{\Lambda^{N}} \prod_{i=1}^{N} \mathrm{~d}^{2} z_{i} w\left(z_{i}, \bar{z}_{i}\right) \prod_{i<j}\left|\mathrm{e}^{z_{i} / R_{c}}-\mathrm{e}^{z_{j} / R_{c}}\right|^{\Gamma},
$$

where $w(z, \bar{z})$ is the one-particle Boltzmann factor given by

$$
w(z, \bar{z})=w(x)=\frac{1}{4 \pi^{2} R_{c}^{2}} \mathrm{e}^{-\pi n \Gamma\left(x^{2}+x_{2 \pi n R_{c}}^{N-1}\right.} .
$$

Completing the square in the Boltzmann factor of (3.12), shifting the variables $x_{i} \rightarrow x_{i}-\frac{N-1}{4 \pi n R_{c}}$, and then rescaling variables $z_{i} \rightarrow z_{i} / \sqrt{\pi n \Gamma}$ we finally get

$$
\begin{aligned}
Z_{\mathrm{J}}^{(2)}= & \frac{1}{N !} \frac{1}{\left(8 \pi^{3} n \gamma R_{c}^{2}\right)^{N}} \mathrm{e}^{-\frac{\gamma \pi}{8 \pi^{2} n R_{c}^{2}} N(N-1)^{2}} \\
& \times \int_{\Lambda^{N}} \prod_{i=1}^{N} \mathrm{~d}^{2} z_{i} \mathrm{e}^{-x_{i}^{2}} \prod_{i<j}\left|\mathrm{e}^{\sqrt{\frac{1}{2 \pi n \gamma R_{c}^{2}}} z_{i}}-\mathrm{e}^{\sqrt{\frac{1}{2 \pi n R_{c}^{2}}} z_{j}}\right|^{2 \gamma},
\end{aligned}
$$

where we replaced $\Gamma=2 \gamma$ for the general case following [42].

The gauge theory partition function (3.4) is proportional to the partition function of the two-dimensional jellium model (3.14) in the thermodynamic limit $N, L \rightarrow \infty$ with $n$ constant, ${ }^{3}$ for $\gamma=1$ with the identification

$$
\tau=8 \pi n R_{c}^{2},
$$

as

$$
\hat{Z}_{\mathrm{YM}}^{(4)}=\mathrm{e}^{-3 \beta N^{2} / 16 R} \pi^{2 N} \hat{Z}_{\mathrm{J}}^{(2)}(\gamma=1)
$$

The partition function (3.14) is computed for various values of $\gamma$ in [42], and in particular for $\gamma=1$ it takes the form

$Z_{\mathrm{J}}^{(2)}(\gamma=1)=\prod_{j=0}^{N-1}\left(\frac{1}{2 \pi R_{c}} \int_{-L / 2}^{L / 2} \mathrm{~d} x \mathrm{e}^{-2 \pi n x^{2}+(2 j-(N-1)) x / R_{c}}\right)$.

In the thermodynamic limit the integral is Gaussian, and we find

$$
\hat{Z}_{\mathrm{J}}^{(2)}(\gamma=1)=\left(\frac{1}{8 \pi^{2} R_{c}^{2} n}\right)^{N / 2} \mathrm{e}^{\frac{1}{24 \pi R_{c} n} N\left(N^{2}-1\right)} .
$$

${ }^{3}$ In this limit, the integration volume becomes

$$
\int_{\Lambda^{N}} \prod_{i=1}^{N} \mathrm{~d}^{2} z_{i}=2^{N} \int_{\mathbb{R}^{N}} \prod_{i=1}^{N} \mathrm{~d} x_{i} \int_{[0, \pi]^{N}} \prod_{i=1}^{N} \mathrm{~d} y_{i},
$$

where we used the fact that the integrand $\operatorname{Im}\left(z_{i}\right)=y_{i}$ is an even function. 
Via (3.18) we can now compute the large $N$ limit of the partition function (3.4) for the low temperature limit of $\mathcal{N}=4$ supersymmetric Yang-Mills theory at finite weak coupling, and we find

$$
\hat{Z}_{\mathrm{YM}}^{(4)}=\left(\frac{\pi^{3 / 2}}{\sqrt{\tau}}\right)^{N / 2} N ! \mathrm{e}^{-3 \beta N^{2} / 16 R+N\left(N^{2}-1\right) / 3 \tau} .
$$

The partition function (3.19) is identical, up to a proportionality factor $2^{N}$, to the partition function (3.7), and therefore the free energy in the large $N$ limit reads

$$
\hat{F}_{\mathrm{YM}}^{(4)}=\left(\frac{3}{16}-\frac{\lambda}{12 \pi^{2}}\right) \frac{N^{2} \beta}{R}+\mathcal{O}(N),
$$

which coincides with (3.9).

In the Coulomb gas description of [16], the low temperature distribution of the eigenvalues lies uniformly in a band of width $2 A$ and circumference $\pi$. This is consistent with the two-dimensional jellium picture on a cylinder of length $L$ and circumference $2 \pi R_{c}$ : The identification [8] can be written as

$$
\frac{\beta \lambda}{2 \pi^{2} R}=\frac{L}{2 R_{c}}=2 A,
$$

in agreement with the result of [16]. This coincidence can be substantiated by noticing that the interpretation of the gauge theory effective action (3.1) as a Coulomb gas in an external potential considered in [16] is in fact the twodimensional jellium model. This is already apparent in (3.1), where the term $\log \sinh \left|z_{i}-z_{j}\right|$ corresponds to the interaction potential between the charge carriers, the term $x_{i}^{2}$ is related to the charged particle-background interaction, and the $z_{i}$-independent term of order $N^{2}$ is proportional to the background-background interaction constant $B_{N}$ [42]. Hence the external potential in the Coulomb gas picture is the background-background interaction in the twodimensional jellium description.

\section{E. Dimensional reduction}

We shall now study the dimensional reduction of the Laughlin wave function on the cylinder and the thin cylinder limit of the two-dimensional jellium system. In both cases we end up with the Coulomb gas description of the Chern-Simons matrix model.

In [43] one can find an explicit relationship between the Laughlin state of the quantum Hall effect and certain one-dimensional exactly solvable models with long-range interactions such as the Calogero model and the Sutherland model. In the limit of a strong magnetic field $B \rightarrow \infty$, the charge carriers in two dimensions are constrained to the lowest Landau level and two of the four phase space degrees of freedom freeze, reducing the number of effective degrees of freedom to two, one in space representation and one in momentum representation. Depending on the two-dimensional geometry of the Hall system, the onedimensional representation of the Laughlin ground state corresponds to the ground state of either the Calogero model (for the disk) or the Sutherland model (for the cylinder); in the latter case the axial degrees of freedom freeze (in space representation) [43]. However, instead of the axial degrees of freedom one can also dually reduce the periodic degrees of freedom by working in momentum representation. The Laughlin ground state with filling factor $p$ on the cylinder is of the form (3.5) with the change of coordinates $z_{j} \rightarrow z_{j} / \sqrt{B}$ and restoring the $B$ dependence on $\gamma_{B}=\frac{\ell_{B}}{R_{c}}=1 / \sqrt{B} R_{c}$ in units where $\hbar=e=1$. Then the one-dimensional reduction in the momentum representation of the Laughlin state on a cylinder is given by [43]

$$
\begin{aligned}
\left\langle t_{1}, \ldots, t_{N} \mid \Psi_{N}\right\rangle= & \prod_{i=1}^{N} \exp \left(-\frac{1}{2 B} \frac{\partial^{2}}{\partial t_{i}^{2}}\right) \\
& \times \mathrm{e}^{-B t_{i}^{2} / 2} \prod_{j<k}\left(\mathrm{e}^{t_{j} / R_{c}}-\mathrm{e}^{t_{k} / R_{c}}\right)^{p},
\end{aligned}
$$

where $t_{i}$ is the eigenvalue of the eigenstate $\left|t_{i}\right\rangle$ of the operator $X_{i}=x_{i}+\Pi_{y_{i}} / B$ for the guiding center coordinate of the cyclotron motion. ${ }^{4}$ In the limit of strong magnetic field $B \rightarrow \infty$, this wave function reduces to

$$
\prod_{i=1}^{N} \mathrm{e}^{-B\left(t_{i}-t_{0}\right)^{2}} \prod_{j<k}\left(\sinh \left(\frac{2\left(t_{j}-t_{k}\right)}{R_{c}}\right)\right)^{p},
$$

where $t_{0}=p(N-1) / 2 B R_{c}$. As in Sec. II G, this is the wave function of a one-dimensional model with interaction potential $\sinh ^{-2}\left(x_{i}-x_{j}\right)$. An intriguing consequence of the strong magnetic field limit is that the wave function of the one-component plasma in one dimension with this interaction potential for filling factor $p=1$ is related to the Chern-Simons matrix model (1.3), as shown by [15]. ${ }^{5}$

Using the Laughlin wave function interpretation of the low temperature limit of supersymmetric Yang-Mills theory in four dimensions, we can apply the strong magnetic field limit to the expression (3.7). This suggests that we should identify the magnetic field $B$ with the quantity $\tau$ so that

$$
B=\tau=\frac{4 \pi^{2} N R T}{\lambda}
$$

Therefore the strong magnetic field limit corresponds to $\tau \gg 1$. We should then take into account the domain of

\footnotetext{
${ }^{4}$ Our notation differs from that of [43], where $x_{i}$ denote the periodic coordinates and $y_{i}$ the axial coordinates.

${ }^{5}$ An alternative perspective on the relationship between the Chern-Simons gauge theory on $S^{3}$ and the Sutherland model can be found in [44].
} 
validity of the effective action (3.1) from [16], which is determined at the weak 't Hooft coupling $\lambda$ via one-loop perturbation theory [30]. There it was argued that the perturbative calculation is valid for the range of temperatures with

$$
0 \leq R T \ll \frac{1}{\lambda}
$$

at weak coupling $\lambda$. This range is satisfactory for high temperatures because the radius of the spatial sphere $S^{3}$ provides a natural infrared cutoff of order $R \sim 1 / \sqrt{\lambda} T$. However, there is no restriction on $R T$ for low temperatures. From (3.24) it follows that the large $\tau$ limit is valid only for low temperatures of order of $\lambda$, i.e. $R T \gtrsim \lambda$ at large $N$, and it might break down for low temperatures of order $R T \ll \lambda$.

The geometrical meaning of the strong magnetic field limit can be deduced in momentum representation where the axial degrees of freedom on the cylinder are kept and the periodic ones are frozen [43]. In this description, the two boundaries of the cylindrical Laughlin droplet are placed at $X_{1}=0$ and $X_{2}=p(N-1) / B R_{c}$. Taking $B \rightarrow \infty$ requires sending $R_{c} \rightarrow 0$ so that $X_{2}$ is constant and the cylinder does not collapse to a circle. Thus the strong magnetic field limit freezes the radial degrees of freedom reducing the geometry of the cylinder effectively to one dimension.

\section{ACKNOWLEDGMENTS}

We thank Sabine Jansen for correspondence on Coulomb gases. The work of G. G. was partially supported by the A. G. Leventis Foundation and the A. S. Onassis Public Benefit Foundation Grant No. F-ZG 097/ 2010-201. G. G. thanks the Department of Mathematical Analysis, Universidad Complutense de Madrid for the kind hospitality during his visit. The work of R J.S. was partially supported by the Consolidated Grant No. ST/J000310/1 from the UK Science and Technology Facilities Council, and by Grant No. RPG-404 from the Leverhulme Trust. The work of M. T. has been partially funded by the Project Entanglement in Quantum Systems (MTM2011-26912), the Project QUITEMAD: QUantum Information TEchnologies MADrid (S2009/ESP-1594), and a Juan de la Cierva Fellowship.
[1] E. Witten, Conformal Field Theory in Four and Six Dimensions, edited by U. Tillmann, Topology, Geometry and Quantum Field Theory, Proceedings of the 2002 Oxford Symposium in Honour of the 60th Birthday of Graeme Segal (Cambridge University Press, Cambridge, England, 2004), pp. 405-420.

[2] M. R. Douglas, J. High Energy Phys. 02 (2011) 1.

[3] N. Lambert, C. Papageorgakis, and M. SchmidtSommerfeld, J. High Energy Phys. 01 (2011) 083.

[4] S. Bolognesi and K. Lee, Phys. Rev. D 84, 106001 (2011).

[5] I. R. Klebanov and A. A. Tseytlin, Nucl. Phys. B 475, 164 (1996).

[6] M. Henningson and K. Skenderis,J. High Energy Phys. 07 (1998) 023.

[7] H.-C. Kim and S. Kim, J. High Energy Phys. 05 (2013) 144.

[8] J. Kallen, J. A. Minahan, A. Nedelin, and M. Zabzine, J. High Energy Phys. 10 (2012) 184.

[9] J. Kallen, J. Qiu, and M. Zabzine, J. High Energy Phys. 08 (2012) 157.

[10] J. Kallen and M. Zabzine, J. High Energy Phys. 05 (2012) 125.

[11] N. Kurokawa and S. Koyama, Forum mathematicum 15, 839 (2003).

[12] M. Marino, Commun. Math. Phys. 253, 25 (2005).

[13] R. Gopakumar and C. Vafa, Adv. Theor. Math. Phys. 2, 413 (1998).

[14] R. J. Szabo and M. Tierz, Nucl. Phys. B 876, 234 (2013).

[15] M. Tierz, Mod. Phys. Lett. A 24, 3157 (2009).
[16] S. A. Hartnoll and S. P. Kumar, Phys. Rev. D 76, 026005 (2007).

[17] M. Tierz, Mod. Phys. Lett. A 19, 1365 (2004).

[18] R. J. Baxter, Math. Proc. Cambridge Philos. Soc. 59, 779 (1963)

[19] M. Kashiwara, Commun. Math. Phys. 133, 249 (1990).

[20] P. J. Forrester, J. Stat. Phys. 56, 767 (1989).

[21] M. F. Atiyah, Topology 29, 1 (1990).

[22] L. C. Jeffrey, Commun. Math. Phys. 147, 563 (1992).

[23] J. A. Minahan, A. Nedelin, and M. Zabzine, J. Phys. A 46, 355401 (2013).

[24] Y. Dolivet and M. Tierz, J. Math. Phys. (N.Y.) 48, 023507 (2007).

[25] J. P. Gauntlett, D. Martelli, J. Sparks, and D. Waldram, Adv. Theor. Math. Phys. 8, 711 (2004).

[26] J. Qiu and M. Zabzine, arXiv:1307.3149.

[27] T. Nishioka, Y. Tachikawa, and M. Yamazaki, J. High Energy Phys. 08 (2011) 003.

[28] M. Girardeau, J. Math. Phys. (N.Y.) 1, 516 (1960).

[29] E. H. Lieb and W. Liniger, Phys. Rev. 130, 1605 (1963).

[30] T. J. Hollowood, S. P. Kumar, and A. Naqvi, J. High Energy Phys. 01 (2007) 001.

[31] E. H. Rezayi and F. D. M. Haldane, Phys. Rev. B 50, 17199 (1994).

[32] A. Seidel, H. Fu, D.-H. Lee, J. M. Leinaas, and J. E. Moore, Phys. Rev. Lett. 95, 266405 (2005).

[33] A. Seidel and D.-H. Lee, Phys. Rev. Lett. 97, 056804 (2006). 
[34] E. J. Bergholtz and A. Karlhede, J. Stat. Mech. 2006, L04001.

[35] E. J. Bergholtz, J. Kailasvuori, E. Wikberg, T. H. Hansson, and A. Karlhede, Phys. Rev. B 74, 081308(R) (2006).

[36] S. Jansen, E. H. Lieb, and R. Seiler, Commun. Math. Phys. 285, 503 (2009).

[37] S. de Haro and M. Tierz, Nucl. Phys. B 731, 225 (2005).

[38] C. Beasley and E. Witten, J. Diff. Geom. 70, 183 (2005).
[39] R. B. Laughlin, Phys. Rev. Lett. 50, 1395 (1983).

[40] D. J. Thouless, Surf. Sci. 142, 147 (1984).

[41] Ph. Choquard, P. J. Forrester, and E. R. Smith, J. Stat. Phys. 33, 13 (1983).

[42] L. Samaj, J. Wagner, and P. Kalinay, J. Stat. Phys. 117, 159 (2004).

[43] H. Azuma and S. Iso, Phys. Lett. B 331, 107 (1994).

[44] R. J. Szabo and M. Tierz, J. Phys. A 43, 265401 (2010). 